\title{
Measurement of the branching fraction ratio
}

$\mathscr{B}\left(B_{c}^{+} \rightarrow \psi(2 S) \pi^{+}\right) / \mathscr{B}\left(B_{c}^{+} \rightarrow J / \psi \pi^{+}\right)$

\section{Liupan An*}

On behalf of the LHCb collaboration

Tsinghua University

E-mail: liupan.an@cern.ch

Using the $p p$ collision data collected by LHCb at center-of-mass energies $\sqrt{s}=7 \mathrm{TeV}$ and $8 \mathrm{TeV}$, corresponding to an integrated luminosity of $3 \mathrm{fb}^{-1}$, the ratio of the branching fraction of the $B_{c}^{+} \rightarrow \psi(2 S) \pi^{+}$decay relative to that of the $B_{c}^{+} \rightarrow J / \psi \pi^{+}$decay is measured to be $0.268 \pm 0.032$ (stat) \pm 0.007 (syst) $\pm 0.006(\mathrm{BF})$. The first uncertainty is statistical, the second is systematic, and the third is due to the uncertainties on the branching fractions of the $J / \psi \rightarrow \mu^{+} \mu^{-}$ and $\psi(2 S) \rightarrow \mu^{+} \mu^{-}$decays. This measurement is consistent with the previous LHCb result, and the statistical uncertainty is halved.

16th International Conference on B-Physics at Frontier Machines 2-6 May 2016

Marseille, France

${ }^{*}$ Speaker. 


\section{Introduction}

In the Standard Model of particle physics the $B_{c}$ meson family is unique because it contains two different heavy flavor quarks, charm and beauty. The ground state of the $B_{c}$ meson family has a rich set of decay modes since either constituent quark can decay with the other as a spectator, or they can annihilate to a virtual $W$ boson. The search for new $B_{c}^{+}$decay channels ${ }^{1}$ and precise measurements of their branching fractions can improve the understanding of Quantum Chromodynamics (QCD). The $B_{c}^{+} \rightarrow \psi(2 S) \pi^{+}$decay was first observed with $p p$ collision data collected by LHCb $[1,2]$ at a center-of-mass energy of $\sqrt{s}=7 \mathrm{TeV}$, corresponding to an integrated luminosity of $1.0 \mathrm{fb}^{-1}$ [3]. The ratio of the branching fraction of the $B_{c}^{+} \rightarrow \psi(2 S) \pi^{+}$decay with respect to that of the $B_{c}^{+} \rightarrow J / \psi \pi^{+}$decay, defined as

$$
R_{\mathscr{B}} \equiv \frac{\mathscr{B}\left(B_{c}^{+} \rightarrow \psi(2 S) \pi^{+}\right)}{\mathscr{B}\left(B_{c}^{+} \rightarrow J / \psi \pi^{+}\right)}
$$

was measured to be $0.250 \pm 0.068$ (stat) \pm 0.014 (syst) \pm 0.006 (BF). The result is dominated by the statistical uncertainty. The analysis presented here [4] updates the previous LHCb measurement using the full $p p$ collision data collected by LHCb in 2011 and 2012 at $\sqrt{s}=7 \mathrm{TeV}$ and $8 \mathrm{TeV}$ respectively, corresponding to a total integrated luminosity of $3 \mathrm{fb}^{-1}$. Due to the increased data sample and an improved analysis method, the statistical uncertainty is reduced by half, allowing a more powerful test of the theories.

\section{Analysis strategy}

The relative branching fraction can be calculated as

$$
\frac{\mathscr{B}\left(B_{c}^{+} \rightarrow \psi(2 S) \pi^{+}\right)}{\mathscr{B}\left(B_{c}^{+} \rightarrow J / \psi \pi^{+}\right)}=\frac{\mathscr{B}\left(B_{c}^{+} \rightarrow \psi(2 S) \pi^{+}, \psi(2 S) \rightarrow \mu^{+} \mu^{-}\right)}{\mathscr{B}\left(B_{c}^{+} \rightarrow J / \psi \pi^{+}, J / \psi \rightarrow \mu^{+} \mu^{-}\right)} \times \frac{\mathscr{B}\left(J / \psi \rightarrow \mu^{+} \mu^{-}\right)}{\mathscr{B}\left(\psi(2 S) \rightarrow \mu^{+} \mu^{-}\right)} .
$$

Assuming lepton universality, the $J / \psi \rightarrow \mu^{+} \mu^{-}$and $\psi(2 S) \rightarrow \mu^{+} \mu^{-}$branching fractions can be substituted with the more precisely measured ones in the $e^{+} e^{-}$channel [5]. The first term can be calculated as

$$
\frac{N\left(B_{c}^{+} \rightarrow \psi(2 S) \pi^{+}, \psi(2 S) \rightarrow \mu^{+} \mu^{-}\right)}{N\left(B_{c}^{+} \rightarrow J / \psi \pi^{+}, J / \psi \rightarrow \mu^{+} \mu^{-}\right)} \times \frac{\varepsilon\left(B_{c}^{+} \rightarrow J / \psi \pi^{+}, J / \psi \rightarrow \mu^{+} \mu^{-}\right)}{\varepsilon\left(B_{c}^{+} \rightarrow \psi(2 S) \pi^{+}, \psi(2 S) \rightarrow \mu^{+} \mu^{-}\right)},
$$

where $N$ is the signal yield, and $\varepsilon$ is the efficiency. During the trigger stage, the $B_{c}^{+} \rightarrow \psi(2 S) \pi^{+}$ or $B_{c}^{+} \rightarrow J / \psi \pi^{+}$candidates are required to have a muon track with a high transverse momentum and of good quality, and the invariant mass of the muon pair should be greater than $2.95 \mathrm{GeV} / c^{2}$. A loose pre-selection is applied offline, followed by a Boosted Decision Tree (BDT) selection [6, 7] to further separate signal from background. The BDT classifier uses information on the candidate's kinematic properties, decay length, vertex quality, impact parameter and angle between the particle momentum and the vector from the primary to the secondary vertex as input, and outputs a response value which indicates how much the candidate is like a signal. The distributions of the variables that are used in the BDT are similar for $B_{c}^{+} \rightarrow J / \psi \pi^{+}$and $B_{c}^{+} \rightarrow \psi(2 S) \pi^{+}$decays. The BDT threshold cut is chosen to maximize the $B_{c}^{+} \rightarrow \psi(2 S) \pi^{+}$signal significance, with which more than $99 \%$ of the background is rejected.

\footnotetext{
${ }^{1}$ Charge conjugation is implied.
} 

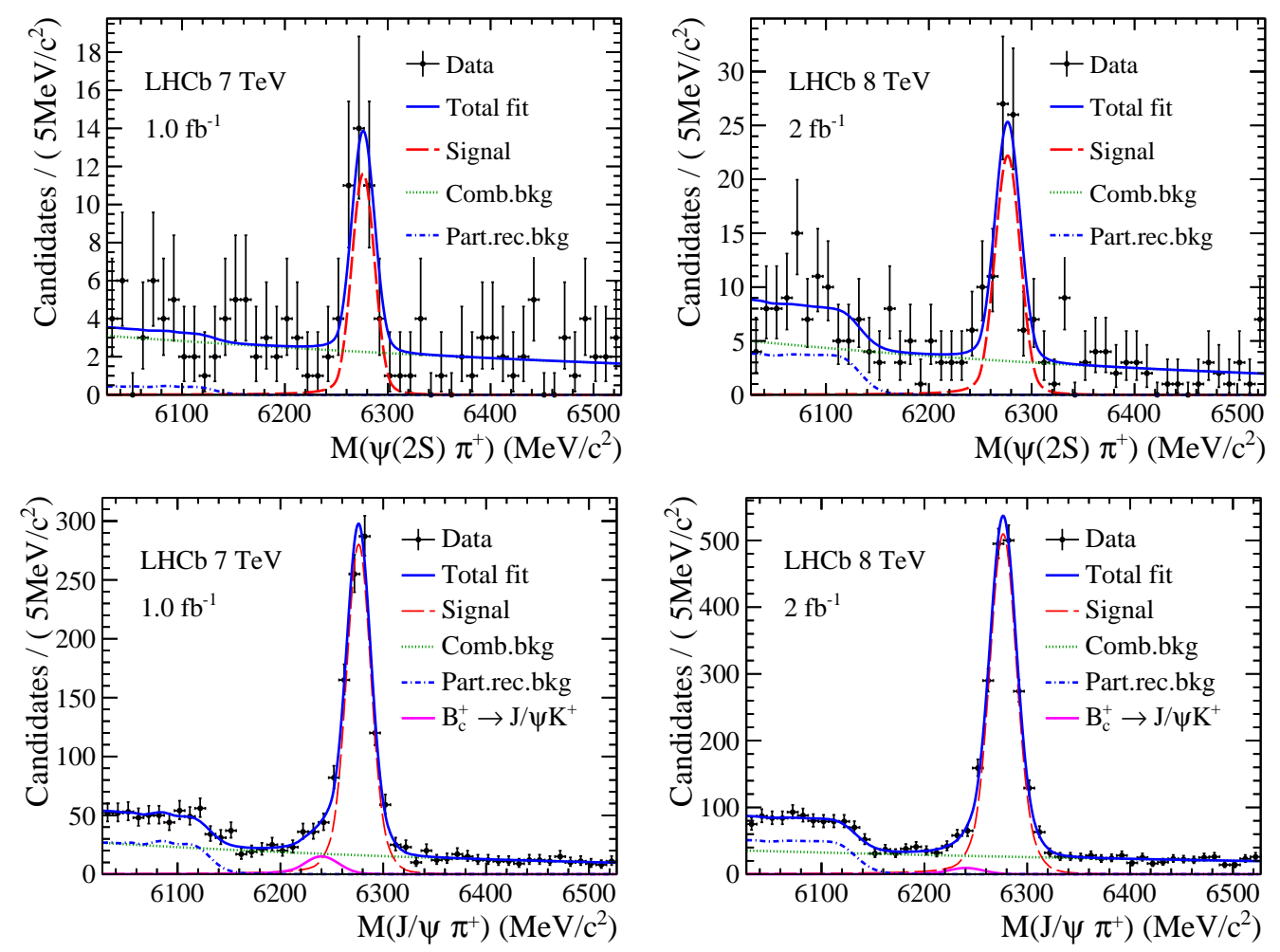

Figure 1: Fit to the reconstructed $B_{c}^{+}$mass distribution for (top) $B_{c}^{+} \rightarrow \psi(2 S) \pi^{+}$and (bottom) $B_{c}^{+} \rightarrow J / \psi \pi^{+}$ using (left) 2011 and (right) 2012 data samples. Black points with error bars represent the data, and the various fitting components are indicated in the legend.

\section{Signal yields and efficiencies}

The signal yields are extracted from unbinned extended maximum likelihood fits to the invariant mass distributions of $\psi(2 S) \pi^{+}$or $J / \psi \pi^{+}$in the range $[6027,6527] \mathrm{MeV} / c^{2}$, as shown in Figs. 1 for 2011 and 2012 data respectively. The signal probability density functions are modelled by double-sided Crystal Ball functions [8] with tail parameters determined from simulation; the combinatorial backgrounds are described with an exponential function; and the partially reconstructed backgrounds are modelled by simulated shape. For the $B_{c}^{+} \rightarrow J / \psi \pi^{+}$channel, there is contamination from $B_{c}^{+} \rightarrow J / \psi K^{+}$with $K^{+}$misidentified as $\pi^{+}$, which is modelled by a double-sided Crystal Ball function with parameters determined from simulation. All efficiencies are determined using simulated samples. To account for any discrepancy between data and simulation, the particle identification efficiencies are calibrated using a $\pi^{+}$sample from $D^{*}$-tagged $D^{0} \rightarrow K^{-} \pi^{+}$decays [9]. Due to the use of the same final state for the normalization, most of the efficiencies cancel.

\section{Systematic uncertainties}

Several sources of systematic uncertainty on the $R_{\mathscr{B}}$ measurement are studied and are summarized in Table 1. The largest contribution comes from the background modelling. It is estimated by reducing the fit range to $[6164,6527] \mathrm{MeV} / \mathrm{c}^{2}$ to exclude the partially reconstructed background. 
Table 1: Summary of systematic uncertainties on the $R_{\mathscr{B}}$ measurement.

\begin{tabular}{lc}
\hline Component & Uncertainty \\
\hline Signal shape & $0.6 \%$ \\
Background shape & $2.4 \%$ \\
BDT classifier & $0.2 \%$ \\
Monte-Carlo Statistics & $0.3 \%$ \\
Trigger efficiency & $1.1 \%$ \\
$B_{c}^{+}$lifetime & $0.1 \%$ \\
\hline Total & $2.7 \%$ \\
\hline
\end{tabular}

\section{Results}

In summary, using the $3 \mathrm{fb}^{-1} p p$ collision data collected by LHCb at center-of-mass energies $\sqrt{s}=7 \mathrm{TeV}$ and $8 \mathrm{TeV}$, the relative branching fraction of the $B_{c}^{+} \rightarrow \psi(2 S) \pi^{+}$decay to the $B_{c}^{+} \rightarrow$ $J / \psi \pi^{+}$decay is measured to be $0.268 \pm 0.032$ (stat) \pm 0.007 (syst) $\pm 0.006(\mathrm{BF})$. The result is consistent with the previous LHCb result $0.250 \pm 0.068$ (stat) \pm 0.014 (syst) \pm 0.006 (BF) [3]. This measurement is in agreement with the predictions of non-relativistic QCD at next-to-leading order $\left(0.26_{-0.06}^{+0.05}\right)[10]$ and perturbative QCD based on $k_{\mathrm{T}}$ factorization $\left(0.29_{-0.11}^{+0.17}\right)$ [11].

\section{References}

[1] LHCb collaboration, A. A. Alves Jr. et al., The LHCb detector at the LHC, JINST 3 (2008) S08005.

[2] LHCb collaboration, R. Aaij et al., LHCb detector performance, Int. J. Mod. Phys. A30 (2015) 1530022, [arXiv:1412.6352].

[3] LHCb collaboration, R. Aaij et al., Observation of the decay $B_{c}^{+} \rightarrow \psi(2 S) \pi^{+}$, Phys. Rev. D 87 (2013) 071103(R), [arXiv:1303.1737].

[4] LHCb collaboration, R. Aaij et al., Measurement of the branching fraction ratio $\mathscr{B}\left(B_{c}^{+} \rightarrow \psi(2 S) \pi^{+}\right) / \mathscr{B}\left(B_{c}^{+} \rightarrow J / \psi \pi^{+}\right)$, Phys. Rev. D 92 (2015) 072007, [arXiv: 1507.03516 ]

[5] Particle Data Group, K. A. Olive et al., Review of Particle Physics, Chin. Phys. C 38 (2014) 090001.

[6] L. Breiman, J. H. Friedman, R. A. Olshen, and C. J. Stone, Classification and regression trees, Wadsworth international group, Belmont, California, USA, 1984.

[7] R. E. Schapire and Y. Freund, A decision-theoretic generalization of on-line learning and an application to boosting, Jour. Comp. and Syst. Sc. 55 (1997) 119.

[8] T. Skwarnicki, A study of the radiative cascade transitions between the Upsilon-prime and Upsilon resonances, PhD thesis, Institute of Nuclear Physics, Krakow, 1986, DESY-F31-86-02.

[9] A. Powell et al., Particle identification at LHCb, POS ( ICHEP 2010) 020.

[10] C.-F. Qiao, P. Sun, D. Yang, and R.-L. Zhu, $B_{c}$ exclusive decays to charmonia and light mesons in QCD factorization at next-to-leading order accuracy, Phys. Rev. D 89 (2014) 034008, [arXiv:1209.5859].

[11] Z. Rui et al., The $B_{c} \rightarrow \psi(2 S) \pi, \eta_{c}(2 S) \pi$ decays in the perturbative QCD approach, Eur. Phys. J. C 75(6) (2015) 293, [arXiv:1505.02498]. 